\title{
THE DIMENSION SUBALGEBRA PROBLEM FOR ENVELOPING ALGEBRAS OF LIE SUPERALGEBRAS
}

\author{
DAVID M. RILEY
}

(Communicated by Kenneth Goodearl)

\begin{abstract}
Let $L$ be an arbitrary Lie superalgebra over a field of characteristic different from 2 . Denote by $\omega u(L)$ the ideal generated by $L$ in its universal enveloping algebra $U(L)$. It is shown that $L \cap \omega u(L)^{n}=\gamma_{n}(L)$ for each $n \geq 1$, where $\gamma_{n}(L)$ is the $n$th term of the lower central series of $L$. We also prove that $\omega u(L)$ is a residually nilpotent ideal if and only if $L$ is residually nilpotent. Both these results remain true in characteristic 2 provided we take $L$ to be an ordinary Lie algebra.
\end{abstract}

\section{INTRODUCTION}

Let $L=L_{0} \oplus L_{1}$ be a Lie superalgebra over a field $K$ of characteristic $p \neq 2$. In the case $p=2$, let $L$ denote an ordinary Lie algebra. We shall write $U(L)$ for the enveloping algebra of $L$, and we let $\omega u(L)$ denote the augmentation ideal of $U(L)$ generated by $L$. When $p=3$, we add to the axioms of a Lie superalgebra the axiom $[[y, y], y]=0$ for all $y \in L_{1}$ to ensure that we can embed $L$ in $U(L)$. The primary problem we wish to address here is the characterisation of the dimension subalgebras

$$
D_{n}(L)=L \cap \omega u(L)^{n} \quad(n \geq 1)
$$

without reference to the ring-structure of $U(L)$. These subalgebras are the analogues of the dimension subgroups of a group. Let $R G$ denote the group ring of a group $G$ over a commutative ring $R$ with identity, and let $\Delta_{R}(G)$ denote its augmentation ideal. The dimension subgroups of $G$ over $R$ are defined to be the subgroups

$$
D_{n}(G)=G \cap\left(1+\Delta_{R}(G)^{n}\right) \quad(n \geq 1) .
$$

The characterisation of these subgroups is known as the dimension subgroup problem. Jennings [J] and Lazard [L1] determined exact expressions for these subgroups when $R$ is a field. Although considerable progress has been in the dimension subgroup problem in the case $R=\mathbb{Z}$, there is still no complete solution. The 'integral dimension subgroup conjecture' was that $D_{n}(G)$ is merely

Received by the editors November 15, 1993 and, in revised form, March 16, 1994.

1991 Mathematics Subject Classification. Primary 16S30, 17A70, 17B35, 17 B70.

The author would like to thank the University of Alberta for its hospitality while this research was carried out. 
the $n$th term of the lower central series of $G$. This, however, was disproved long ago by Rips in [Ri]. We intend to prove here that dimension subalgebras of Lie superalgebras over fields are particularly well behaved. Let $\gamma_{n}(L)$ denote the $n$th term of the lower central series of $L$.

Theorem 1.1. Let $K$ be a field of characteristic $p \geq 0$. If $p \neq 2$, let $L$ be any Lie superalgebra over $K$. If $p=2$, let $L$ be any ordinary Lie algebra over $K$. Then $D_{n}(L)=\gamma_{n}(L)$ for all $n \geq 1$.

In the study of group rings, many questions have been solved by using the dimension subgroups of $G$. Also, in [RS], Shalev and the present author studied several aspects of restricted enveloping algebras by first characterising the dimension subalgebras of restricted Lie algebras. Perhaps the use of dimension subalgebras of Lie superalgebras will prove to be useful as well.

Our techniques will allow us to deduce the following theorem. Its group ring-theoretic counterpart was proved by Hartley in $[\mathrm{H}]$.

Theorem 1.2. Let $L$ be as above. Then $\omega u(L)$ is a residually nilpotent ideal if and only if $L$ is residually nilpotent.

We shall deduce also the analogue of a theorem of Lazard [L2] and Quillen [Q] about graded group rings. Let $\operatorname{gr}(U(L))$ denote the graded associative algebra $\bigoplus_{m>0} \omega u(L)^{m} / \omega u(L)^{m+1}$, and let $\operatorname{gr}(L)$ represent the associated graded Lie superalgebra of $L$ defined by $\operatorname{gr}(L)=\bigoplus_{m \geq 1} \gamma_{m}(L) / \gamma_{m+1}(L)$.

Theorem 1.3. Let $L$ be as above. Then $\operatorname{gr}(U(L)) \cong U(\operatorname{gr}(L))$ as graded associative superalgebras.

It must be mentioned that Knus [K] proved Theorems 1.1 and 1.3 in the case that $L$ is a finite-dimensional Lie algebra over a field of characteristic zero, and McConnell [Mc] proved Theorem 1.2-also in the finite-dimensional Lie algebra situation. See [R] and [RS] for the restricted Lie-theoretic analogues of Theorems 1.1-1.3.

\section{Preliminaries}

The reader is referred to the paper [BP] of Bergen and Passman for a thorough presentation of the definition of a Lie superalgebra. We adopt their basic terminology here. Thus, let $\sigma$ denote the $K$-linear automorphism of $L$ that induces the $\mathbb{Z}_{2}$-grading on $L$ and, hence, on $U(L)$.

We shall require the Poincaré-Birkhoff-Witt Theorem for enveloping algebras of Lie superalgebras. See Scheunert [S], for example.

Poincaré-Birkhoff-Witt Theorem. Let $\mathscr{B}_{0}$ and $\mathscr{B}_{1}$ be totally ordered bases of $L_{0}$ and $L_{1}$, respectively. Then $U(L)$ has a $K$-basis of all ordered monomials $b_{1}^{\alpha_{1}} b_{2}^{\alpha_{2}} \cdots b_{s}^{\alpha_{s}} c_{1} c_{2} \cdots c_{t}$, such that $b_{1}<b_{2}<\cdots<b_{s}$ in $\mathscr{B}_{0}, c_{1}<c_{2}<\cdots<c_{t}$ in $\mathscr{B}_{1}$, and $\alpha_{i}$ are non-negative integers.

\section{Filtrations AND DimENSION SUbALGEBRAS}

Let $L$ be a Lie superalgebra over a field $K$ of characteristic $p \neq 2$. A $\sigma$-filtration of $\omega u(L)$ is a descending sequence of $\sigma$-stable ideals of $U(L)$

$$
\omega u(L)=E_{(1)} \supseteq E_{(2)} \supseteq \cdots \supseteq E_{(n)} \supseteq \cdots
$$


satisfying $E_{(i)} E_{(j)} \supseteq E_{(i+j)}$ for all $i, j$. For example, it is not difficult to see that the sequence of powers of $\omega u(L),\left\{\omega u(L)^{i}\right\}$, is a $\sigma$-filtration of $\omega u(L)$. Any such sequence gives rise naturally to a sequence of subspaces of $L$ :

$$
L=L_{(1)} \supseteq L_{(2)} \supseteq \cdots \supseteq L_{(n)} \supseteq \cdots
$$

by defining $L_{(n)}=L \cap E_{(n)}$. It is a routine matter to verify that these subspaces are $\sigma$-stable and satisfy the condition $\left[L_{(i)}, L_{(j)}\right] \subseteq L_{(i+j)}$ for all $i, j$. We call any such descending sequence of subspaces of $L$ satisfying these two properties a $\sigma$-filtration of $L$. Clearly then, the lower central series of $L$ is an example of a $\sigma$-filtration of $L$, as is the sequence of the dimension subalgebras of $L$.

We have just seen that a $\sigma$-filtration of $\omega u(L)$ gives rise to a $\sigma$-filtration of $L$. In fact the converse is also true. Suppose that $\left\{L_{(n)}\right\}$ is any $\sigma$-filtration of $L$. For each homogeneous $x \in L$ we define the height of $x$, which we shall denote by $\nu(x)$, to be the largest subscript $n$ such that $x \in L_{(n)}$ if $n$ exists, and to be infinite if it does not. Now for each integer $m \geq 1$, let $E_{(m)} \subseteq U(L)$ be the $K$-linear span of all the products of the form

$$
x_{1} x_{2} \cdots x_{l}
$$

for some $l$, where $x_{1}, x_{2}, \ldots, x_{l}$ are homogeneous elements of $L$ such that $\nu\left(x_{1}\right)+\cdots+\nu\left(x_{l}\right) \geq m$. Since each of the monomials $x_{1} x_{2} \cdots x_{l}$ lies in either $U(L)_{0}$ or $U(L)_{1}$, it is clear that $E_{(m)}$ is $\sigma$-stable and hence that the sequence $\left\{E_{(n)}\right\}$ is a $\sigma$-filtration of $\omega u(L)$.

The close relationship between $\left\{L_{(n)}\right\}$ and $\left\{E_{(n)}\right\}$ is demonstrated in the following proposition.

Proposition 3.1. Let $\left\{L_{(n)}\right\}$ be a $\sigma$-filtration of a Lie superalgebra $L$ over a field $K$ of characteristic different from 2 , and let $\mathscr{B}_{0}$ and $\mathscr{B}_{1}$ be totally ordered bases of $L_{0}$ and $L_{1}$, respectively, chosen so that

$$
L_{(n)}=\left\langle b \in \mathscr{B}_{0}, c \in \mathscr{B}_{1} \mid \nu(b) \geq n, \nu(c) \geq n\right\rangle_{K} .
$$

Write $\left\{E_{(n)}\right\}$ for the filtration of $\omega u(L)$ induced by $\left\{L_{(n)}\right\}$. Then the following statements hold for each $n \geq 1$.

(1) The set of all monomials of the form $b_{1}^{\alpha_{1}} b_{2}^{\alpha_{2}} \cdots b_{s}^{\alpha_{s}} c_{1} c_{2} \cdots c_{t}$ with the properties that $\sum_{i=1}^{s} \alpha_{i} \nu\left(b_{i}\right)+\sum_{j=1}^{t} \nu\left(c_{j}\right) \geq n, b_{1}<\cdots<b_{s}, c_{1}<$ $\cdots<c_{t}, 0 \leq \alpha_{i}$ for each $i$, and $s, t \geq 1$ form a basis of $E_{(n)}$ over $K$.

(2) $L_{(n)}=L \cap \bar{E}_{(n)}$.

Proof. Let $E_{(n, k)}$ be the $K$-linear span of all the products of the form $y_{1} \cdots y_{l}$, where $y_{1}, \ldots, y_{l}$ are homogeneous elements of $L, \sum_{j=1}^{l} \nu\left(y_{j}\right) \geq n$, and $l \leq$ $k$. Let $G$ be the subgroup of permutations $\sigma$ of the permutation group $\mathscr{S}_{k}$ on $\{1,2, \ldots, k\}$ that satisfy

$$
y_{\sigma(1)} y_{\sigma(2)} \cdots y_{\sigma(k)} \in K^{*} y_{1} y_{2} \cdots y_{l}+E_{\left(\sum_{j=1}^{k} \nu\left(y_{j}\right), k-1\right)}
$$

for all homogeneous $y_{1}, \ldots, y_{k} \in L$.

Claim 1. $G=\mathscr{S}_{k}$.

Indeed, let $\tau \in \mathscr{S}_{k}$ satisfy $\tau(i)=i+1, \tau(i+1)=i$, and $\tau(j)=j$ for all $j \notin\{i, i+1\}$. Then

$$
\begin{aligned}
y_{\tau(1)} \cdots y_{\tau(k)} & =y_{1} \cdots y_{i+1} y_{i} \cdots y_{k} \\
& =y_{1} \cdots y_{k}+y_{1} \cdots y_{i-1}\left(y_{i+1} y_{i}-y_{i} y_{i+1}\right) y_{i+2} \cdots y_{k} .
\end{aligned}
$$


Consider two cases. First, suppose that $y_{i}$ or $y_{i+1}$ lies in $L_{0}$. Then $y_{i+1} y_{i}-$ $y_{i+1} y_{i}=\left[y_{i+1}, y_{i}\right]$, so that

$$
y_{1} \cdots y_{i-1}\left[y_{i+1}, y_{i}\right] y_{i+2} \cdots y_{k} \in E_{\left(\sum_{j=1}^{k} \nu\left(y_{j}\right), k-1\right)}
$$

since $\nu\left(\left[y_{i}, y_{i+1}\right]\right) \geq \nu\left(y_{i}\right)+\nu\left(y_{i+1}\right)$. Next assume that both $y_{i}$ and $y_{i+1}$ lie in $L_{1}$. Then $y_{i+1} y_{i}=\left[y_{i+1}, y_{i}\right]-y_{i} y_{i+1}$, so that

$$
\begin{aligned}
y_{\tau(1)} \cdots y_{\tau(k)} & =-y_{1} \cdots y_{k}+y_{1} \cdots y_{i-1}\left[y_{i+1}, y_{i}\right] y_{i+2} \cdots y_{k} \\
& \in-y_{1} \cdots y_{k}+E_{\left(\sum_{j=1}^{k} \nu\left(y_{j}\right), k-1\right)} .
\end{aligned}
$$

Therefore $\tau \in G$. The claim now follows from the fact that $\mathscr{S}_{k}$ is generated by elementary transpositions.

Claim 2. $E_{(n, k)}$ is spanned by the set of monomials $b_{1}^{\alpha_{1}} b_{2}^{\alpha_{2}} \cdots b_{s}^{\alpha_{s}} c_{1} c_{2} \cdots c_{t}$, where $\sum_{i=1}^{s} \alpha_{i} \nu\left(b_{i}\right)+\sum_{j=1}^{t} \nu\left(c_{j}\right) \geq n, b_{1}<\cdots<b_{s}, c_{1}<\cdots<c_{t}, 0 \leq \alpha_{i}$ for each $i$, and $t+\sum_{i=1}^{s} \alpha_{i} \leq k$.

By definition these elements are contained in $E_{(n, k)}$. We prove that they span $E_{(n, k)}$ by induction on $k \geq 1$. The case $k=1$ follows by our choice of basis:

$$
E_{(n, 1)}=\left\langle b \in L_{0}, c \in L_{1} \mid \nu(b) \geq n, \nu(c) \geq n\right\rangle_{K}=L_{n} .
$$

Assume now that the claim holds for all $j<k$. Let homogeneous $y_{1}, \ldots, y_{k} \in$ $L$ satisfy $\sum_{j=1}^{k} \nu\left(y_{j}\right) \geq n$. Since $y_{1} \cdots y_{k-1} \in E_{\sum_{j=1}^{k-1} \nu\left(y_{j}\right), k-1}$, by the induction hypothesis we find that $y_{1} \cdots y_{k-1}$ is a linear combination of monomials $b_{1}^{\alpha_{1}} b_{2}^{\alpha_{2}} \cdots b_{s}^{\alpha_{s}} c_{1} c_{2} \cdots c_{t}$, where $b_{1}<\cdots<b_{s}, c_{1}<\cdots<c_{t}, 0 \leq \alpha_{i}$ for each $i$, $\sum_{i=1}^{s} \alpha_{i} \nu\left(b_{i}\right)+\sum_{j=1}^{t} \nu\left(c_{j}\right) \geq \sum_{j=1}^{k-1} \nu\left(y_{j}\right)$, and $t+\sum_{i=1}^{s} \alpha_{i} \leq k-1$. Also note that $y_{k}$ is spanned by the $b \in \mathscr{B}_{0}$ and $c \in \mathscr{B}_{1}$ with height at least $\nu\left(y_{k}\right)$.

Now consider two situations. First suppose that $b$ appears in the representation of $y_{k}$. Without loss of generality, we may assume that $b=b_{i}$, say. Then by the first claim

$$
b_{1}^{\alpha_{1}} b_{2}^{\alpha_{2}} \cdots b_{s}^{\alpha_{s}} c_{1} c_{2} \cdots c_{t} b_{i} \in K^{*} b_{1}^{\alpha_{1}} \cdots b_{i}^{\alpha_{i}+1} \cdots b_{s}^{\alpha_{s}} c_{1} c_{2} \cdots c_{t}+E_{(n, k-1)} .
$$

Secondly suppose that $c$ appears in the representation of $y_{k}$. Then either $c$ does not appear in the representation of $y_{1} y_{2} \cdots y_{k-1}$ or $c=c_{j}$, say. In the latter case

$$
b_{1}^{\alpha_{1}} b_{2}^{\alpha_{2}} \cdots b_{s}^{\alpha_{s}} c_{1} c_{2} \cdots c_{t} c_{j} \in K^{*} b_{1}^{\alpha_{1}} b_{2}^{\alpha_{2}} \cdots b_{s}^{\alpha_{s}} c_{1} \cdots c_{j}^{2} \cdots c_{t}+E_{(n, k-1)} .
$$

Since $c_{j}^{2}=\frac{1}{2}\left[c_{j}, c_{j}\right]$, we may replace $c_{j}^{2}$ by a linear combination of elements from $\mathscr{B}_{0}$ and $\mathscr{B}_{1}$ with height at least $2 \nu\left(c_{j}\right)$. The resulting representation lies in $E_{(n, k-1)}$. Therefore, in every case, we find that $y_{1} \cdots y_{k}$ is spanned by the monomials $b_{1}^{a_{1}} b_{2}^{a_{2}} \cdots b_{s}^{a_{s}} c_{1} c_{2} \cdots c_{t}$ modulo $E_{(n, k-1)}$. The claim now follows from the induction hypothesis.

Because $E_{(n)}=\sum_{k \geq 1} E_{(n, k)}$, it is clear from the second claim that the monomials in question span $E_{(n)}$. The linear independence of the monomials follows from the PBW Theorem for enveloping algebras of Lie superalgebras. Hence we have proven part (1).

Part (2) follows readily from part (1). 
Let us remark that the proposition above also holds for Lie algebras over fields of characteristic 2. Also, let us point out that we may now define the weight of a monomial $b_{1}^{\alpha_{1}} b_{2}^{\alpha_{2}} \cdots b_{s}^{\alpha_{s}} c_{1} c_{2} \cdots c_{t}$ by $\sum_{i=1}^{s} \alpha_{i} \nu\left(b_{i}\right)+\sum_{j=1}^{t} \nu\left(c_{j}\right)$.

\section{Proofs of THE THEOREMS}

We now exploit Proposition 3.1.

Proof of Theorem 1.1. Let us write $\left\{L_{(n)}\right\} \leq\left\{H_{(n)}\right\}$ for two $\sigma$-filtrations of $L$ if $L_{(n)} \subseteq H_{(n)}$ for all $n \geq 1$. Certainly then $\left\{\gamma_{n}(L)\right\}$ is the unique smallest $\sigma$ fifltration of $L$. Let $\left\{E_{(n)}\right\}$ be the $\sigma$-filtration of $\omega u(L)$ induced by $\left\{\gamma_{n}(L)\right\}$. Because $E_{(1)}=\omega u(L)$, we have $\omega u(L)^{n}=E_{(1)}^{n} \subseteq E_{(n)}$. Hence,

$$
D_{n}(L)=L \cap \omega u(L)^{n} \subseteq L \cap E_{(n)}=\gamma_{n}(L)
$$

by part (2) of the proposition above. Consequently, $D_{n}(L)=\gamma_{n}(L)$ for all $n \geq 1$.

Let us next point out that the $\sigma$-filtration induced by $\left\{\gamma_{n}(L)\right\}$ is in fact $\left\{\omega u(L)^{n}\right\}$. Indeed, from the proof above, it remains only to show that $E_{(n)} \subseteq$ $\omega u(L)^{n}$ for each $n$. Let $\mathscr{B}_{0}$ and $\mathscr{B}_{1}$ be bases of $L_{0}$ and $L_{1}$ chosen as in Proposition 3.1 with respect to $\left\{\gamma_{n}(L)\right\}$. Let $y \in E_{(n)}$. Then by part (1) of the proposition we can write $y$ uniquely as the linear combination of monomials of the form $b_{1}^{\alpha_{1}} b_{2}^{\alpha_{2}} \cdots b_{s}^{\alpha_{s}} c_{1} c_{2} \cdots c_{t}$, where each $b_{i} \in \mathscr{B}_{0}$, each $c_{j} \in \mathscr{B}_{1}, b_{1}<\cdots<$ $b_{s}, c_{1}<\cdots<c_{t}$, each $\alpha_{i} \geq 0$, and weight $\sum_{i=1}^{s} \alpha_{i} \nu\left(b_{i}\right)+\sum_{j=1}^{t} \nu\left(c_{j}\right) \geq n$. But $b_{i} \in \gamma_{\nu\left(b_{i}\right)}(L) \subseteq \omega u(L)^{\nu\left(b_{i}\right)}$ and $c_{j} \in \gamma_{\nu\left(c_{j}\right)}(L) \subseteq \omega u(L)^{\nu\left(c_{j}\right)}$, so that $y \in \omega u(L)^{n}$, as required.

Proof of Theorem 1.2. It is clear that the residual nilpotence of $\omega u(L)$ forces the residual nilpotence of $L$; so it suffices to prove the converse. Suppose then, to the contrary, that $\bigcap_{n>1} \gamma_{n}(L)=0$, but $y$ is a non-trivial element of $\bigcap_{n \geq 1} \omega u(L)^{n}$. Let $\mathscr{B}_{0}$ and $\mathscr{B}_{1}$ be bases of $L_{0}$ and $L_{1}$ chosen as in Proposition 3.1 with respect to $\left\{\gamma_{n}(L)\right\}$. According to the discussion above, this $\sigma$-filtration of $L$ induces the canonical $\sigma$-filtration $\left\{\omega u(L)^{n}\right\}$. By the PBW Theorem for enveloping algebras of Lie superalgebras, we can write $y$ uniquely as the linear combination of monomials of the form $b_{1}^{\alpha_{1}} b_{2}^{\alpha_{2}} \cdots b_{s}^{\alpha_{s}} c_{1} c_{2} \cdots c_{t}$, where each $b_{i} \in \mathscr{B}_{0}$, each $c_{j} \in \mathscr{B}_{1}, b_{1}<\cdots<b_{s}, c_{1}<\cdots<c_{t}$, and each $\alpha_{i} \geq 0$. Then, by part (1) of Proposition 3.1, the weight of each of the monomials in the representation of $y$ must be infinite for otherwise $y \notin \omega u(L)^{n+1}$, where $n$ is the least of these finite weights. But, by assumption, $L$ has no non-zero elements of infinite height. Clearly this leads us to the desired contradiction.

Put $\gamma_{\omega}(L)=\bigcap_{n>1} \gamma_{n}(L)$. Obviously, $\gamma_{\omega}(L)$ is congruent to 0 modulo all the powers of $\omega u(L)$, which means that $\operatorname{gr}(U(L)) \cong \operatorname{gr}(U(\bar{L}))$ where $\bar{L}=$ $L / \gamma_{\omega}(L)$. Also $\operatorname{gr}(L) \cong \operatorname{gr}(\bar{L})$.

Proof of Theorem 1.3. From the preceding remarks, we see that we may assume that $\gamma_{\omega}(L)=0$. Consider now the natural injection $\phi: \operatorname{gr}(L) \rightarrow \operatorname{gr}(U(L))$. If we consider $\operatorname{gr}(U(L))$ as a Lie superalgebra, then $\phi$ is a monomorphism. Let $\psi: U(\operatorname{gr}(L)) \rightarrow \operatorname{gr}(U(L))$ denote its unique extension to a homomorphism of associate algebras. Choose bases $\mathscr{B}_{0}$ and $\mathscr{B}_{1}$ of $L_{0}$ and $L_{1}$ as in Proposition 3.1 with respect to $\left\{\gamma_{n}(L)\right\}$. Then $\left\{b+\gamma_{\nu(b)+1}(L) \mid b \in \mathscr{B}_{0}\right\}$ 
and $\left\{c+\gamma_{\nu(b)+1}(L) \mid c \in \mathscr{B}_{1}\right\}$ are bases of $\operatorname{gr}(L)_{0}$ and $\operatorname{gr}(L)_{1}$, respectively. Put $\bar{b}=b+\gamma_{\nu(b)+1}(L)$ and $\bar{c}=c+\gamma_{\nu(c)+1}(L)$ for each $b \in \mathscr{B}_{0}$ and $c \in \mathscr{B}_{1}$. Then, by the PBW Theorem for enveloping algebras of Lie superalgebras, the set of all monomials $\bar{b}_{1}^{\alpha_{1}} \bar{b}_{2}^{\alpha_{2}} \cdots \bar{b}_{s}^{\alpha_{s}} \bar{c}_{1} \bar{c}_{2} \cdots \bar{c}_{t}$ such that each $b_{i} \in \mathscr{B}_{0}$, each $c_{j} \in \mathscr{B}_{1}$, $b_{1}<\cdots<b_{s}, c_{1}<\cdots<c_{t}$, and each $\alpha_{i} \geq 0$ forms a basis $\mathscr{G}$ of $U(\operatorname{gr}(L))$. But, by applying Proposition 3.1, we see that $\psi(\mathscr{G})$ is a basis for $\operatorname{gr}(U(L))$. Hence $\psi$ is an isomorphism.

\section{REFERENCES}

[BP] J. Bergen and D. S. Passman, Delta methods in enveloping algebras of Lie superalgebras, Trans. Amer. Math. Soc. 334 (1992), 259-280.

[H] B. Hartley, The residual nilpotence of wreath products, Proc. London Math. Soc. 2 (1970), 365-392.

[J] S. A. Jennings, The group ring of a class of infinite nilpotent groups, Canad. J. Math. 7 (1955), 169-187.

[K] M. A. Knus, On the enveloping algebra and the descending central series of a Lie algebra, J. Algebra 12 (1969), 335-338.

[L1] M. Lazard, Sur les groupes nilpotents et les anneaux de Lie, Ann. Sci. École Norm. Sup. 71 (1954), 101-190.

[L2] _ Groupes analytiques p-adiques, Inst. Hautes Études Sci. Publ. Math. 26 (1965).

[Mc] J. C. McConnell, The intersection theorem for a class of noncommutative rings, Proc. London Math. Soc. 17 (1967), 487-498.

[Q] D. G. Quillen, On the associated graded ring of a group ring, J. Algebra 10 (1968), 411-418.

[R] D. M. Riley, Numbers of generators of ideals in graded group rings and p-adic analytic pro-p groups of dimension one, Arch. Math. 63 (1994), 402-406.

[RS] D. M. Riley and A. Shalev, Restricted Lie algebras and their envelopes, Canad. J. Math. (to appear).

[Ri] E. Rips, On the fourth integral dimension subgroup, Israel J. Math. 12 (1972), 342-346.

[S] M. Scheunert, The theory of Lie superalgebras, Lecture Notes in Math., vol. 716, SpringerVerlag, Berlin, 1979.

Department of Mathematics, The University of Alabama, Tuscaloosa, Alabama 35487

E-mail address: driley@gp.as.ua.edu 